\title{
BRAF V600 Wild Type
}

National Cancer Institute

\section{Source}

National Cancer Institute. BRAF V600 Wild Type. NCI Thesaurus. Code C134468.

A genetic finding indicating that codon 600 of the BRAF gene encodes the wild type amino acid, valine. 